\title{
La minga como imaginario social. Una mirada a la pedagogía de resiliencia indígena en Colombia ${ }^{1}$
}

\author{
La minga as social imagery, A regard to the \\ indian resilience pedagogy in Colombia \\ O mutirão como imaginário social. Uma abordagem da \\ Pedagogia de Resiliência Indígena na Colômbia
}

Recepción: 30/03/2020

José Pascual Mora García ${ }^{2}$

Universidad de Cundinamarca

Grupo de investigación HISULA - UPTC

https://orcid.org/0000-0002-5345-6808

José del Carmen Correa Alfonso ${ }^{3}$

Universidad de Cundinamarca, Colombia

https://orcid.org/0000-0002-5957-0564

Evaluación: 28/04/2020

Aceptación: 28/05/2020

Artículo de Investigación - Revisión

https://doi.org/10.19053/01227238.10355

\section{RESUMEN}

El presente trabajo tiene por objetivo abordar la minga como una de las manifestaciones sociales enmarcadas en la cultura de la resistencia indígena en Colombia. La propuesta es original por cuanto recupera para la historia de la educación una práctica ancestral, expresión de una actividad pedagógica colectiva que forma parte de las enseñanzas de numerosos pueblos indígenas andinos

de Colombia que se reúnen para levantar edificaciones, preparar o cultivar terrenos, o recolectar cosechas; en nuestro caso, queremos presentarla como una forma de resistencia social, de defensa de sus derechos democráticos y de presentación de iniciativas frente al Acuerdo de Paz. Metodológicamente, es una investigación documental que se fundamenta en las epistemologías del sur y la tradición de la

$1 \quad$ Artículo de revisión, proyecto de investigación Pedagogías, Paz y Poblaciones resilientes (HISULA - Universidad Pedagógica y Tecnológica de Colombia UPTC / Universidad de Cundinamarca UDEC), liderado por la Dra. Diana Soto Arango, y los coinvestigadores José Pascual Mora García y José del Carmen Correa Alfonso.

2 Doctorado en innovación y sistema educativo por la Universidad UNIVERSITAT ROVIRA I VIRGILI. Doctor en Historia Económica y Social de Venezuela de la Universidad Santa María en Venezuela. Magíster en Gerencia Educativa de la Universidad Nacional Experimental del Táchira, Licenciado en Filosofía de la Universidad Central de Venezuela. Profesor emérito de la Universidad de los Andes, Estado Táchira Venezuela. Actualmente es investigador del Doctorado en Ciencias de la Educación de la Universidad de Cundinamarca. Profesor pionero de la Red RUDECOLOMBIA desde 1998. Coordinador Fundador desde el año 2000 de grupo de investigación HEDURE. Fue presidente de la Sociedad de Historia de la Educación Latinoamericana SHELA entre el año 2011 al 2016. Correo electrónico: pascualmoraster@gmail.com / jpascualmora@ucundinamarca.edu.co

3 Administrador de Empresas de la Universidad de Cundinamarca, Magíster en Administración de Empresas -MBA de la Universidad Externado de Colombia, Magíster en Gestión de la Calidad de la Educación Superior de la Universidad de Pamplona y Doctor en Educación de la Universidad de la Salle de Costa Rica. Investigador Junior. Correo electrónico:jcorrea@ ucundinamarca.edu.co 
Escuela de los Annales, los historiadores marxistas británicos y las pedagogías alternativas ${ }^{4}$. De todo lo cual se concluye que la recuperación de los imaginarios ancestrales a través de la minga hace que sea posible potenciar la Pedagogía de Resiliencia para la Paz.

Palabras clave: pedagogía; resiliencia; modernidad alternativa; minga, paz.
The current work aims at addressing one of the social manifestations in the culture of indigenous resistance in Colombia, such as the Minga. The proposal is original meanwhile an ancestral practice is recovered for the history of education. The minga as an expresión of a collective pedagogical practice is a part of the teachings of a large part of the Andean indigenous peoples of Colombia, and that merge to build, prepare or cultivate land or collect crops, and in our case, we want to present the demonstration as a form of social resistance, of their democratic rights and actions against the Peace Agreement. Methodologically, it is a desk-based research that is epistemologically focused on the epistemologies of the south and the tradition of the French Annalist School, British Marxist historians and Alternative Pedagogies (MoraGarcía, 2019). From which it is concluded that the recovery of ancestral imaginaries through La Minga allows us to promote the Pedagogy of Resilience for Peace.

Key words: Pedagogy, resilience, alternative modernity, minga, peace.

\section{RESUMO}

O presente trabalho tem por objetivo abordar uma das manifestações sociais na cultura de resistência indígena na Colômbia, como é a Minga. A proposta é original, enquanto resgata para a história da educação uma prática ancestral. A Minga como expressão de uma prática pedagógica coletiva faz parte dos ensinamentos da grande parte de povos indígenas andinos da Colômbia, e que se fundiram para construir edificações, preparar ou cultivar terrenos ou colheita de safras, e em nosso caso, queremos apresentar a manifestação como forma de resistência social, de seus direitos democráticos e iniciativas frente ao Acordo de Paz. Metodologicamente é uma pesquisa documental que se fundamenta epistemologicamente nas epistemologias do sul e da tradição da Escola dos Annales francesa, nos historiadores marxistas britânicos e nas Pedagogias Alternativas (Mora-García, 2019). Deste estudo se conclui que a recuperação dos imaginários ancestrais através da Minga nos permite potencializar a Pedagogia de Resiliência para a Paz.

Palavras-chave: Pedagogia; Resiliência; Modernidade Alternativa; Minga; Paz.

\section{INTRODUCCIÓN}

En Colombia, la historiografía - atravesada por diferentes enfoques - ha sido especialmente abundante en el tema de la cosmovisión indígena; el indigenismo y sus efectos en las políticas de Estado. Desde el siglo XIX ha insistido en

4 José Pascual Mora García, “Aproximación a las pedagogías alternativas: de la pedagogía de la diversidad a las pedagogías de la resiliencia en el marco del postacuerdo", Revista Historia de la Educación Colombiana vol. 22, n. ${ }^{\circ} 22$ (2019). https://doi.org/10.22267/ rhec. 192222.51 
la importancia de los movimientos sociales de carácter étnico, pero no necesariamente como una reivindicación de las "culturas indígenas" 5 .

En este sentido tenemos que diferenciar entre la comprensión intelectual de la etnicidad y las diversas formas de invención de la tradición ${ }^{6}$. Históricamente, las élites fueron los ejes protagónicos en su dimensión operativa al gobierno, funcionaron como aparatos ideológicos del Estado y sirvieron como base del diseño del tiempo histórico nacional; organizaron los ritos, los imaginarios y conmemoraciones cívicas, la historiografía y la ensayística, incluso las obras literarias. Así, por ejemplo, Brubaker ${ }^{7}$ denomina "etnicidad como cognición" a la forma como algunos no indígenas conciben la categoría étnica y, en particular, a las culturas indígenas. Amselle ${ }^{8}$, destaca que algunos sectores de la cultura dominante utilizan la construcción de un campo intelectual o bien para hablar en nombre de los pueblos originarios, o para expresar los intereses de las élites indígenas y que muchas veces han servido para legitimar la dominación. En esa comprensión intelectual de la etnicidad recordamos al romanticismo del siglo XIX, que

[...] es una corriente de pensamiento que tuvo gran influencia en el indigenismo. A semejanza de Europa, el romanticismo en Colombia consistió en una "recuperación" de las tradiciones, así como una exaltación del carácter nacional (o local en ciertos casos) de las culturas (...) El indigenismo de las élites colombianas, de hecho, comenzó a nacer en este contexto intelectual marcado por tendencias europeas (Langebaek, 2003, pp. 78-79) ${ }^{9}$.

Estas tendencias intelectuales tuvieron efectos también en corrientes artísticas y culturales en Colombia. A manera de ejemplos, podemos mencionar la pieza de teatro Sulma de José Joaquín Borda, la cual representaba los rituales del Templo del Sol de los Muiscas en Sogamoso. ${ }^{10}$

La recreación de los imaginarios sociales influenciados por el romanticismo hizo posible la construcción de narraciones inventadas, que entrarían en el orden de lo que Hobsbawm denomina "invención de la tradición", pero que no por eso son carentes de valor histórico e historiográfico, como lo apunta el mismo autor ${ }^{11}$. La invención de tradición responde a alguna función social y sirve para establecer o simbolizar cohesión social o pertenencia a un grupo real o

$5 \quad$ Véanse Christian Gros, Colombia indígena: Identidad cultural y cambio social (Bogotá: CEREC, 1991); María Teresa Findji, "From Resistance to Social Movement: The Indigenous Authorities Movement in Colombia", en The Making of Social Movements in Latin America: Identity, Strategy, and Democracy, ed. Arturo Escobar y Sonia E. Álvarez (New York: Westview Press, 1992); Virginie Laurent, Comunidades indígenas, espacios politicos y movilización electoral en Colombia, 1990-1998. Motivaciones, campos de acción e impactos (Bogotá: Institut français détudes andines, Instituto Colombiano de Antropología e Historia-ICANH, 2005).

6 Eric Hobsbawm y Terence Ranger, eds., La invención de la tradición (Barcelona: Editorial Crítica, 2003).

7 Rogers Brubaker, Ethnicity without groups (Cambridge, Mass: Harvard University Press, 2014).

8 Jean-Loup Amselle, “En nombre de los pueblos: primitivismos y poscolonialismos", Revista Colombiana de Antropología vol. 49, n. 2 (2013).

9 El texto citado remite a Carl Henrik Langebaek R., Arqueología colombiana. Ciencia, pasado y exclusión (Bogotá: Colciencias, 2003).

10 Jean Paul Sarrazin, "La categoría indígena definida desde la hegemonía y sus alcances en la institucionalidad colombiana", Justicia, n. 32 (2017): 146. Doi: https://doi.org/10.17081/just.23.32.2909 [cursivas del original].

11 Hobsbawm y Ranger, La invención de la tradición. 
artificial. Por eso también ha servido para recrear mitos fundacionales, es decir, construir representaciones con un primer origen vinculado con raíces míticas o apellidos de nobleza declarada; buscan dar legitimidad y fundamento a jerarquías sociales y relaciones de autoridad, como por ejemplo el patriarcalismo o el clasismo. Invenciones destinadas a inculcar en el inconsciente colectivo creencias, sistemas de valores y códigos convencionales de conducta. Esas narraciones son recuperadas desde "[...] la década de 1850, [cuando] surgen nuevos intentos de fundar la identidad nacional en la imagen del indio del pasado. De hecho, bajo la influencia del romanticismo, diversos autores escribieron dramas de la Conquista, contando la suerte de los reyes Muisca e imaginando un gran reino indígena"12. Armando Suescún recrea en su obra Derecho y sociedad (tomo I) ${ }^{13}$, ese pasado mítico en la mitología Muisca. Aunque Marquardt ${ }^{14}$ cuestiona esa mirada, es necesario reivindicar ese pensamiento alterno que recupera imaginarios sociales que han sobrevivido en la historia lenta en nuestras raíces indígenas milenarias y que resistieron a la destrucción occidental.

La importancia del estudio de la arqueología de los imaginarios sociales es importante para el estudio del campo intelectual de las comunidades científicas ${ }^{15}$; "Estas ideas románticas que glorificaban al indio de un pasado más bien imaginario, circulaban al mismo tiempo que los indígenas de carne y hueso eran menospreciados, considerados como los representantes de una civilización en decadencia ${ }^{16}$. Luego de la Constitución de 1886, redactada bajo un régimen conservador, los indígenas, vistos como "salvajes" o "medio civilizados", estaban bajo la tutela de las misiones católicas y, desde el punto de vista legal, eran considerados "menores"17.

Desde el punto de vista de la polarización política también se puede observar la rivalidad entre las dos tendencias, liberales y conservadores.

Luego de varios gobiernos conservadores entre 1886 y 1930, el partido liberal ganó las elecciones en 1930, marcando el comienzo del "Régimen Liberal" que se extendió hasta 1946. Este "Régimen" promovió la consolidación de varias instituciones, principalmente la "Escuela Normal Superior", relacionadas con la formación y la investigación en Ciencias Sociales, desarrollando así el interés científico por las comunidades indígenas. Es así que, en 1938, tuvo lugar la Exposición Arqueológica y Etnológica en Bogotá. Esta exposición permitió a los bogotanos de la época - una

12 Ibíd.

13 Armando Suescún M., Derecho y sociedad en la historia de Colombia: El derecho chibcha. Siglo IX - Siglo XVI (Tunja: Universidad Pedagógica y Tecnológica de Colombia, 1998).

14 Bernd Marquardt, Historia del derecho de Hispanoamérica: En perspectiva transnacional y socio-cultural (Bogotá: Casa Editorial Ibáñez, 2019).

15 Félix Báez-Jorge, “Antropología e indigenismo en Latinoamérica: señas de identidad”, en Motivos de la antropología americanista. Indagaciones en la diferencia, coord. Miguel León-Portilla (México D.F: Fondo de Cultura Económica, 2001), 424-444.

16 Roberto Pineda Camacho, "La Constitución de 1991 y la perspectiva del multiculturalismo en Colombia", Revista Alteridades vol. 7, n. ${ }^{\circ} 14$ (1997): 113.

17 Roberto Pineda Camacho, "Estado y pueblos indígenas en el siglo XX. La política indigenista entre 1886 y 1991", Revista Credencial Historia, n. ${ }^{\circ} 146$ (2016); Sarrazin, "La categoría indígena", 147. 
población mayoritariamente "blanca", lo que no era el caso de todas las regiones del país - conocer a los indígenas y sus obras culturales, tales como danzas, músicas y objetos (Perry, 2009) ${ }^{18}$. A semejanza de las exposiciones "universales" realizadas en Europa en la misma época, indígenas vivos eran llevados y observados por los "blancos" como si fuesen curiosidades o animales de zoológico. ${ }^{19}$

El reconocimiento de las comunidades indígenas desde la mirada a la historia constitucional colombiana es una conquista de la Constitución de 1991. En el reciente seminario sobre Teoría de la Justicia y la Constitución (Unilibre, Pereira $)^{20}$ desarrollamos una mirada sinóptica a propósito del Centenario de las Constituciones Sociales de Weimar (1919) y Querétaro (1917), al que le agregamos los aportes de la Constitución colombiana de 1863, la reforma de 1936 y las reformas sociales hasta la Constitución de 1991. En especial destacamos cómo aparece el denominado indigenismo institucionalizado en el texto constitucional, es decir, el momento en que ciertas ideas a propósito de lo indígena, promulgadas por académicos o las comunidades, pudieron ser incorporadas a los discursos e instituciones estatales; los pueblos indígenas como categoría en el discurso constitucional colombiano es una conquista del siglo XX. Como bien lo demuestra Jorge Patiño ${ }^{21}$, la Constitución de Tunja reconoce la ciudadanía a todos los mayores de quince años, en la que se supone que serían asimilados los indígenas, pero al introducir la restricción de tener un "oficio honesto", que se mantengan por sí; genera una discriminación de facto. No se reconoció la condición de comunidades indígenas, sino que se les asimiló a la racionalidad emergente del imaginario de la independencia, en la cual pasaron a ser nuevamente excluidos de sus raíces culturales ancestrales:

[...] la Constitución de Tunja estipuló que "para el nombramiento de electores pueden votar todos los vecinos que pasando de quince años, tengan un oficio honesto, de que se mantengan por sí, y no tengan la tacha que se han expresado para los representantes." (secc. 7-7) (...) Igualmente, determinó que "la universalidad de los ciudadanos constituye el pueblo soberano" (sesión preliminar, cap. 1-19).22

El discurso constitucional se mantuvo sin fundamento étnico, porque el sueño de la independencia buscaba empoderar la clase de los blancos criollos, pero no los indígenas; ya lo decía Simón Bolívar en el Discurso del Congreso de Angostura, el 15 de febrero de 1819: "No somos europeos, no somos indios, sino una especie media entre los aborígenes y los españoles." ${ }^{23}$ Y es que, "efectiva-

18 El texto citado remite a Jean Paul Perry, "La exposición arqueológica y etnográfica de 1938 en Colombia: Un primer intercambio cultural", en Arqueología y etnología en Colombia. La creación de una tradición científica, comp. Carl Henrik Langebaek y Clara Isabel Botero (Bogotá: Universidad de los Andes, Centro de Estudios Socioculturales, 2009), 79-94.

19 Sarrazin, "La categoría indígena", 147.

20 José Pascual Mora, "Mirada sinóptica sobre la Constitución Social a propósito del Centenario de las Constituciones Sociales de Weimar (1919), Querétaro (1917) y el proceso constitucional colombiano”. Heurística, nro. 23 (2019).

21 Jorge Patiño Rojas, República de Tunja 1811-1816. Análisis constitucional (Tunja: Buhos editores Ltda. 2019).

22 Ibíd, 314-315.

23 Simón Bolívar, Obras Completas (Caracas: Pool Reading, 1975), 220 
mente, desde el siglo XIX, Colombia es un país donde se observa un proceso de mestizaje racial y cultural particularmente intenso" 24 .

En la Universidad Nacional es muy relevante el aporte del Grupo de Constitución Comparada liderado por Bernd Marquardt, en el apartado intitulado: "Juridización de los pueblos indígenas: ¿tránsito cultural a occidente?"25. En el mismo se destacan las bondades de la Constitución de 1991 en materia de protección a las comunidades indígenas:

La Constitución de 1991, la jurisprudencia nacional e internacional, y los tratados internacionales de y sobre derechos humanos, han generado una sensación de protección a las comunidades indígenas, mestizas, raizales y afrodescendientes. Esta sensación es producto de la existencia de normas y figuras jurídicas que podrían significar la materialización de la protección a dichas comunidades. ${ }^{26}$

A partir de la Constitución de 1991, a las comunidades indígenas en Colombia se les reconoció en su condición de pueblo; "realizó una transformación en el reconocimiento de la jurisdicción indígena en Colombia haciendo efectivo el reconocimiento de la igualdad en la diversidad, incluyendo la normatividad y la jurisdicción indígena como parte de la normatividad nacional y reconociendo la diversidad étnica como una forma de pluralismo jurídico" ${ }^{27}$. El reconocimiento de la jurisdicción indígena responde a que efectivamente existe una Colombia indígena, según el censo electoral de 2005. Sin embargo, los vacíos entre la articulación de las políticas de Estado y las comunidades indígenas han generado inconsistencias, en el que las comunidades indígenas han tenido que construir una modernidad alternativa, tipificada por

La falta de un diálogo intercultural entre las instituciones del Estado y las comunidades indígenas, (...) los cuales exponen tanto a los grupos de interés económico como a los actores armados ilegales. Es de esta manera como las comunidades indígenas han tenido que fungir como su propio Estado, a través de la práctica de su propio derecho, no desde el respeto constitucional a ellos atribuido, sino desde el estado de necesidad y de apartheid constitucional, que significa no tener otro mecanismo eficaz de protección de sus derechos que las acciones propias y mancomunadas de las comunidades, frente al alto nivel de riesgo en que viven sus tierras, sus costumbres, su integridad y su memoria. ${ }^{28}$

¿Qué significa la minga en el imaginario social indígena colombiano?

24 Sarrazin, "La categoría indígena", 145.

25 Paula Silva Rodríguez y Camilo Ramírez Gutiérrez, "Fórmulas para la paz: eficacia de los derechos humanos en las zonas de violencia y los campos sociales minados por el capitalismo global”, en Paz a través del derecho y de la constitución, coord. Bernd Marquardt, David Ernesto Llinás Alfaro y Aura Helena Peñas Felizzola (Bogotá: Grupo Editorial Ibáñez, 2016).

26 Ibíd., 383.

27 Corte Constitucional de Colombia, Sentencia T-001, Derecho a la diversidad étnica y cultural, 2012, MP J.C. Henao P. Citado por Silva Rodríguez y Ramírez Gutiérrez, "Fórmulas para la paz", 385.

28 Ibíd., 386. 
La minga hace parte de un imaginario social indígena, con una organización que le da forma y espíritu sistemático. La llamada Colombia indígena es un colectivo social que representa

[...] el dos por ciento de la población colombiana de 42 millones de habitantes (que) se han visto afectados desproporcionadamente por oleadas persistentes de violencia y guerra civil. En el año de 2005, alrededor del 30 por ciento de los pueblos indígenas enfrentaba problemas relacionados con el conflicto armado. Entre enero del año 2004 y mayo de 2005 se registraron 299 muertes violentas de indígenas. Los ataques contra las comunidades indígenas continuaron durante 2006 y 2007. En marzo de 2008, la oficina del Alto Comisionado de las Naciones Unidas para los Refugiados (ACNUR) informó que, según varios grupos colombianos de derechos humanos, "muchos grupos indígenas están en eminente peligro de extinción y el peligro más grande proviene de los soldados del gobierno y de los paramilitares respaldados por el ejército, quienes los amenazan, los intimidan, y los acusan de complicidad con los insurgentes, desplazándolos de sus tierras". Los pueblos indígenas son particularmente vulnerables ante los efectos del desplazamiento, puesto que su cultura es especialmente dependiente de la tierra; $[\ldots] .^{29}$

Las comunidades indígenas tienen en la Minga la mejor representación que confirma la existencia de una mentalidad colectiva del espíritu ancestral; apostándole a los andamios geomentales de paz a pesar del etnocidio sistemático, pues

[...] entre el $1^{\circ}$ de enero de 2016 y el 31 de diciembre de 2018 en Colombia fueron asesinados 431 líderes sociales, según la Defensoría del Pueblo. De otro lado, en lo que va corrido del 2019, más de 10 homicidios contra esta población se reporta, de acuerdo con los datos reportados desde diferentes regiones del país. Mientras tanto, la Misión de la ONU en Colombia, hasta la fecha reporta más de 80 excombatientes de las Farc asesinados luego del acuerdo de paz con la antigua guerrilla. ${ }^{30}$

Esa violencia sobre las comunidades indígenas encuentra en las mujeres, uno se los sectores más vulnerables, como es el caso del "asesinato el día 1 de mayo de 2019, de la líder de la comunidad indígena embera chamí, Remelia Aizama Aizama, en San José del Palmar-Chocó. Asesinato vil y cobarde contra una mujer indefensa que se caracterizó por su bondad, respeto y discreción. Defensora de la vida y el territorio" ${ }^{31}$. Las muertes reiteradas de líderes sociales ha sido una constante, como el asesinato de trece líderes de la comunidad Nasa, en los municipios de Toribío, Corinto y Suárez, del Norte del Cauca; "los indígenas están siendo atacados porque los controles territoriales que hace la guardia en

29 Leslie Wirpsa, David Rothschild y Catalina Garzón, “El poder del bastón. La resistencia indígena y la construcción de la paz en Colombia”, en Colombia: La construcción de la paz en tiempos de guerra, ed. Virginia M. Bouvier (Bogotá: Editorial Universidad del Rosario, 2014), 293-315.

30 María Fernanda Quintero Alzate, “Los asesinatos de líderes en Colombia: ¿un tema nacional o internacional?”, América Latina en movimiento, 10 de mayo (2019) https://www.alainet.org/es/articulo/199768 (5/9/2019).

31 Ibíd. 
los resguardos han incomodado y generado malestar en las disidencias" ${ }^{\prime 32}$. Por eso la necesidad de expresar el rechazo ante la subcultura de la muerte en las poblaciones indígenas. Dos hechos recientes nos tocan de cerca en este proceso de la cultura de la muerte a los líderes indígenas, el caso del líder U'wa Joel Aguablanca, quien participó en proyectos del Grupo Hisula liderados por la Dra. Diana Soto Arango, nos hizo sentir de cerca la impotencia ante la violencia gubernamental; al respecto, Diego Naranjo manifestó:

Lamento profundamente la muerte del colega, amigo, educador U'wa Joel Aguablanca Villamizar; no puedo parar de sentir dolor y con la rabia en la garganta, no he hecho otra cosa que compartir en redes sociales la noticia de su asesinato. Es un hecho aterrador que se suma a la muerte de cientos de líderes que trabajan por sus comunidades. En el caso de Joel, coordinador educativo de la nación U'wa, lo recuerdo y siento su pérdida irreparable con más indignación, porque tuve la oportunidad de compartir con él escenarios de diálogo alrededor de la educación intercultural, de las posibilidades que tiene en medio de nuestros contextos tan abrumadores y difíciles. En una de estas reuniones presentó el Proyecto Educativo Comunitario-PEC; un avance significativo en torno a la educación propia de la nación U'wa, su asesinato deja un profundo vacío en quienes trabajamos con él otra educación posible. . $^{33}$

Circunstancia que pone en duda la eficacia de los procesos de paz, sobre todo por el escalamiento de la violencia hacia los líderes sociales, cuyos asesinatos se incrementaron en $53 \%$ respecto del 2019:

El informe lo presentó la Fundación Ideas para la Paz (FIP). Titulado "Dinámicas de la confrontación armada y su impacto humanitario y ambiental", hace un conteo preocupante: 16 líderes fueron asesinados en enero, 11 en febrero, 13 en marzo y nueve en abril, para un total de 49, mientras que en los primeros cuatro meses de 2019 fueron 32. De ahí sale ese 53 \%. Más aún, dice la Fundación, "los departamentos más críticos durante el primer cuatrimestre de 2020 fueron Cauca (con 14 homicidios), Putumayo (6) y Antioquia (5)". Las malas noticias no terminan ahí. Las muertes violentas vienen creciendo especialmente en Tumaco y Buenaventura, territorios en disputa y esenciales para la construcción de un país en paz. Además, la Oficina de la ONU para la Coordinación de Asuntos Humanitarios (OCHA, por sus siglas en inglés) alertó que en los primeros cuatro meses de 2020 crecieron un $5 \%$ los desplazamientos forzados, de los cuales más de la mitad (57\%) fueron masivos. En la Colombia de 2020 todavía hay cientos de personas que tienen que abandonar sus hogares, sus trabajos y sus tierras escapando del riesgo de ser asesinadas. Es una tragedia. ${ }^{34}$

32 Marcela Osorio Granados, "La inversión social también hace parte de la respuesta al Cauca': Óscar Campo", El Espectador, Bogotá, 5 de noviembre, 2019, 4 .

33 Diego Naranjo, El asesinato del líder U'wa, Joel Aguablanca, https://m.facebook.com/story.php?story_fbid=159533815063316 3\&id=100004709794290 (1/6/2020)

34 El Espectador (Editorial): "Los siguen matando, cuando son más necesarios", Bogotá, 28 de mayo, 2020. http://elespectador.com/ opinion/editorial/los-siguen-matando-cuando-son-mas-necesarios-articulo-921437. (30/05/2020). 
En estas condiciones, Boaventura de Sousa Santos propone hablar de democracias de baja intensidad: "vivimos en sociedades de democracia de baja intensidad. El problema pasa por comprender que la democracia es parte del problema, y tenemos que reinventarla si queremos que sea parte de la solución." ${ }^{35}$ Estas democracias se caracterizan por un sujeto que queda alienado de su condición de soberano, enajenando la visión de Rousseau de soberanía popular; "Estamos muy lejos de ese ideal democrático, y por eso hay que ver si podemos crear una contra-hegemonía... tenemos que reinventar la demo-diversidad." ${ }^{\prime 36}$ En esa demo-diversidad caben las comunidades indígenas, ya que tienen una ciudadanía bloqueada, en la medida en que no se le garantizan las condiciones de participación; a pesar de que tienen sus derechos según la Constitución del 91, no se les garantiza sus condiciones materiales. En este escenario los movimientos sociales de la Minga manifiestan esa conciencia social que buscan empoderar las comunidades indígenas históricamente excluidas, entre sus objetivos no solo está el tema hacer valer sus derechos constitucionales sino el exigir el respeto al ser penetrados por la violencia externa; una violencia en la que "han coexistido tres sistemas territoriales, que es necesario modificar: el sistema institucional universal cooptado, que atraviesa fuerte crisis de legitimidad y eficiencia; la justicia indígena más estable; y la 'justicia' ilegal- criminal con sus pactos económicos y botines consigue con el desplazamiento forzoso, las masacres, el robo de tierras y el narcotráfico, recordar las estructuras mentales de la conquista." ${ }^{37}$

Es necesario desmontar el doble estándar a la hora de abordar el tema de los vulnerables, pues todavía está en el inconsciente colectivo la mirada descalificadora; la dialéctica de la negación se impone para descalificar la otredad presente en la Colombia indígena. El ejemplo más patético lo encontramos en la voz de un funcionario del MinInterior, quien salió a la palestra pública tras maltratar fuera de cámara a los indígenas del Cauca: "Yo ya odio a esos hijueputas" (sic), afirmó, luego de que finalizara una reunión virtual entre indígenas del Cauca y funcionarios del MinTIC y del MinInterior; la grabación continuó encendida con micrófonos abiertos y se escucharon las opiniones del funcionario ${ }^{38}$.

Ante estas actitudes nos convencemos más de la necesidad de proyectar un proceso de repedagogización social, inspirado en la Paz Pedagógica ${ }^{39}$ Transmoderna, en donde la transmodernidad implica en la comprensión de Dussel, incorporar nuevas subjetividades que modifiquen la racionalidad social hegemónica; "Una futura cultura trans-moderna, que asume los momentos positivos de la Modernidad (pero evaluados con criterios distintos desde otras culturas milenarias), tendrá una pluriversidad rica y será fruto de un auténtico diálogo intercultural..." ${ }^{40}$ Pensamos que es un gran acierto la mirada que se construye desde la Línea de Investigación Pedagogías Paz y Poblaciones Resilientes en el Doctorado de la Universidad de Cundinamarca,

35 Boaventura de Sousa Santos, Para una democracia de alta intensidad. Renovar la teoría crítica y reinventar la emancipación social. (Buenos Aires: Clacso, 2006), 77

http://bibliotecavirtual.clacso.org.ar/ar/libros/edicion/santos/Capitulo\%20III.pdf (30/05/2020)

36 Ibíd, 78

37 Edgar Revéiz, Cooptación o democracia. La transgresión moral de las élites y el sometimiento de los estados. (Bogotá: Academia colombiana de ciencias económicas, 2015), 71

38 Publimetro Colombia, "Revelan audio de funcionario de MinInterior insultando a indígenas caucanos", 21 de mayo, 2020 https:// www.publimetro.co/co/noticias/2020/05/21/odio-hijueputas-funcionario-mininterior-indigenas-del-cauca.html (22/5/2020).

39 José Pascual Mora García, "La construcción del campo intelectual de la historia de la educación y su aporte a la paz pedagógica: Una mirada interdisciplinaria. Derecho y Realidad, 17(33). 2019 https://doi.org/10.19053/16923936.

40 Enrique Dussel, “Transmodernidad e Interculturalidad. (Interpretación desde la Filosofía de la Liberación)”. 2005. http://red.pucp. edu.pe/ridei/wp-content/uploads/biblioteca/090514.pdf. (30/5/2020), 17 
que se inspira en el Modelo Educativo Digital Transmoderno, que permite incorporar desde la universidad un reconocimiento de las comunidades indígenas.

Aun cuando la Colombia indígena se encuentra institucionalizada, es necesario que la indolencia no se encargue de silenciar estas muertes, por eso es necesario el apoyo a las diversas organizaciones que se asocian en la Organización Nacional Indígena de Colombia (ONIC), entre las que podemos citar: OIA (Antioquia) ${ }^{41}, \mathrm{CRIDEC}$ (Caldas) $^{42}$, CRIR(Risaralda) ${ }^{43}$, ORIQUIN (Quindío) ${ }^{44}$, ORIVAC y ACIVA (Valle del Cauca) ${ }^{45}$, CRIC $(\text { Cauca })^{46}$, CRIHU (Huila) ${ }^{47}$, ASO'UWA (Boyacá, Santander y Norte de Santander) ${ }^{48}$, entre otros ${ }^{49}$.

41 Organización Indígena de Antioquia (OIA), que agrupa, protege y defiende a las comunidades Tule, Zenú (o Sinú) y Embera. "Esta organización nació como consecuencia de las luchas indígenas que se dieron a nivel nacional por la recuperación de tierras, la titulación de los resguardos, la reconstitución de los cabildos y la identidad cultural; en la década del setenta y mediados de los ochenta. Al igual que en el resto del país, en Antioquia, se han organizado para luchar por los derechos de los indígenas y la vigencia de los mismos. En esta lucha han acudido a diferentes mecanismos como las presiones mediante marchas, tomas de oficinas, concertación con el Estado y la participación política, consiguiendo logros significativos, pero también sufriendo por los asesinatos de dirigentes importantes dentro del movimiento. En la presente etapa se valoran los logros obtenidos en la Constitución pero siguen convencidos de que la Organización social es el camino más seguro para la defensa de sus derechos y es por esto que todos los indígenas de Antioquia han dirigido sus acciones hacía la construcción y consolidación de la Organización Indígena de Antioquia (OIA)." http://www.gloobal.net/iepala/gloobal/fichas/ficha.php?entidad=Agentes\&id=1921\&opcion=descripcion

42 El Consejo Regional Indígena de Caldas (CRIDEC) es una organización departamental de derecho propio, filial de la Organización Nacional Indígena de Colombia (ONIC) y su deber social es defender los derechos de las comunidades indígenas de Caldas; agrupa a 17 cabildos indígenas, a saber: resguardo de la Albania (Risaralda-San José), resguardo Cañamomo Lomaprieta (Supía-Riosucio), resguardo Escopetera Pirza (Riosucio-Quinchía, Risaralda), resguardo Nuestra Señora Candelaria de La Montaña (Riosucio), resguardo Totumal (Belalcázar), Territorio Ancestral San Lorenzo (Riosucio-Supía), parcialidad Ambacheke (Palestina), parcialidad Ansea (Anserma), parcialidad Cartama (Marmato), parcialidad Cauromá (Supía), parcialidad Dachi Joma (Anserma), parcialidad El Palmar (Filadelfia), parcialidad La Soledad (Filadelfia), parcialidad La Trina (Supía), asentamiento Bakurukar (Viterbo), asentamiento Dachi Drua (San José), asentamiento Damasco (Neira). La población es de aproximadamente 58000 indígenas del pueblo Embera Chami en su gran mayoría". http://crideccaldas.org/

43 Consejo Regional Indígena de Risaralda.

44 Organización Regional Indígena del Quindío. https://www.elespectador.com/tags/organizacion-regional-indigena-del-quindio-oriquin/

45 Organización Regional Indígena del Valle del Cauca (ORIVAC) y la Asociación de Cabildos Indígenas del Valle del Cauca (ACIVA). https://www.valledelcauca.gov.co/publicaciones/66133/comunidades-indigenas-tendran-un-capitulo-especial--en-el-plan-de-desarrollo-del-valle-del-cauca/

46 El Consejo Regional Indígena del Cauca es una asociación de autoridades indígenas a la cual pertenece el $90 \%$ de los cabildos y comunidades indígenas del departamento del Cauca, Colombia. Fue fundado en Toribío el 24 de febrero de 1971, como una federación de apenas siete cabildos. https://www.google.com/search?q=CRIC+(Cauca)\%2C\&oq=CRIC+(Cauca)\%2C\&aqs=chrome..69i57j017.2313j0j8\&sourceid=chrome\&ie=UTF-8

47 El Consejo Regional Indígena del Huila. "La Resistencia indígena en el departamento se remite a los inicios del siglo XX fundamentando las luchas LIDERADOS POR MANUEL QUINTIN LAME contra la expropiación de las tierras del resguardo ejercidos por la hegemonía del blanco representando por terratenientes, sacerdotes de elite y politiquerías. La población indígena para entonces se reporte en gran número concentrados en los resguardos de: San Antonio de Fortalecillas y del Caugan Dujos, Iquiras, Órganos, Otaseos, Naranjal, La Jagua y otras etnias eran pertenecientes a los pueblos de Tamaz, Nasa, Pijaos, Yanacunas, Andaquíes, entre otros." https://www.crihu.org/p/resena-crihu.html

48 Asociación U'wa. Las luchas se sintetizan en el siguiente petitorio: "El posicionamiento como Pueblo U’wa, son los mismos que venimos presentando en todo este tiempo, han sido y siguen siendo claros, y firmemente los volvemos a reivindicar en este documento: -El gobierno colombiano debe entregarnos los títulos coloniales del resguardo indígena de la nación tuneba otorgados por la corona española. -El respeto irrestricto por parte del Estado colombiano del derecho histórico patrimonial milenario que nos asiste como cultura ancestral, reconociéndonos mediante ley ordinaria o acto administrativo la propiedad absoluta sobre nuestro Resguardo Colonial y Republicano conforme a la teoría del primer ocupante indicado por el Honorable Consejo de Estado en Sentencia del año 1972. -Cancelación inmediata de los títulos mineros otorgados a personas naturales o jurídicas cuya área de adjudicación se encuentre en territorio del Resguardo U'wa. -No a la exploración y explotación de recursos naturales en nuestro Resguardo Unido U'wa y Territorio Ancestral. -No a la Consulta Previa para temas relacionados con explotación de recursos naturales renovables, no renovables y megaproyectos. -Autonomía en la administración territorial del Resguardo U’wa, no aceptamos la coadministración propuesta por Parques Nacionales Naturales de Colombia y Corporaciones Autónomas Regionales". AsoU 'wa. Plan de Salvaguarda Nación U'WA KERA CHIKARA OBIRYACQUINACU. https://siic.mininterior.gov.co/sites/default/files/ pueblo_uwa_boyaca_y_santanderes_-_diagnostico_comunitario.pdf

49 Citaremos las siglas en aras de la economía del lenguaje: UNIPA, ACIESNA y CAMAWARI (Nariño), ASOREWA y FEDEOREWA (Chocó), CRIT (Tolima), ASOPBARI (Norte de Santander), Pueblo Muisca, (Cundinamarca), UNUMA (Meta), CAMAEMKA (Alto Sinú, Córdoba), ASCATIDAR (Arauca), ORIC (Casanare), CRIVI (Vichada), AIPEA, ACITAM, ATICOYA, AZCAITA, OIMA, AZICATCH y ASOAINTAM (Amazonas), CRIOMC y ORUCAPU (Caquetá), ASOCIACIÓN WAYA WAYUU, Organización Wayuu Araurayu, Resguardo de Mayabangloma, Aacigwasug, Painwashi y Yanama (La Guajira), OWYBT (Cesar, Magdalena y 
La minga como fenómeno colectivo ha tenido varios momentos. "Son más de 50 las mingas que el movimiento indígena y campesino del sur del país ha realizado en las últimas dos décadas. Si bien el reclamo de las comunidades ancestrales es de vieja data, solo a partir de 1999, durante el gobierno de Andrés Pastrana, el Estado adquirió compromisos firmados en papel, como el decreto 982 de ese año, que contiene políticas públicas en beneficio de estas comunidades $^{\prime 50}$. Podríamos arriesgar una cronología como propedéutica de las mingas con mayor impacto en la sociedad colombiana:

\begin{abstract}
En septiembre de 2004, setenta mil personas salieron de Santander de Quilichao, un pueblo enclavado en las montañas de los Andes, y caminaron más de noventa kilómetros hacia el norte por la carretera Panamericana, atravesando el departamento del Cauca en el suroccidente de Colombia. Esta ola de mujeres, hombres y niños indígenas, protegidos por cinco mil "guardias indígenas" que portaban bastones ceremoniales (palos adornados con la bandera roja y verde de la etnia nasa), atravesó sin violencia una de las zonas de guerra más complejas de Colombia, con destino a la vibrante ciudad de Cali, poblada por aproximadamente dos millones de personas. Los nasa le dieron a ese evento el nombre de minga, una práctica colectiva tradicional utilizada por gran parte de los ochenta y cinco pueblos indígenas de Colombia para construir edificaciones, preparar o cultivar terrenos o recolectar cosechas. ${ }^{51}$
\end{abstract}

Agregaríamos, la más reciente, ante la negativa de reunirse directamente el presidente Iván Duque; "La Minga2020 ha comenzado su marcha hacia Bogotá, todo parece indicar que, confluirá para el 21 de octubre en el gran Paro Nacional." 52 Esta manifestación nos sirvió de referencia preliminar a nuestro trabajo por ser "una de las movilizaciones indígenas más grandes jamás realizadas, fue representativa de varios siglos de resistencia de los pueblos indígenas colombianos ante la imposición de la violencia y los modelos económicos que amenazan las culturas indígenas, su modo de vida y su misma supervivencia" 53 .

-La movilización del 2008 duró más de sesenta días e incluso llevó a Bogotá a más de cinco mil marchantes ${ }^{54}$.

-La movilización se inició el 12 de octubre de 2013 y finalizó con la firma de acuerdos con el gobierno nacional doce días después, en el resguardo La María (Piendamó, Cauca).

La Guajira), OIK y Pueblo Yupka (Cesar), Pueblo EtteEnaka-Chimila (Magdalena y Cesar), Cabildo Mayor Pueblo Zenú (Córdoba y Sucre), Cabildo Mayor Mokana (Atlántico) y Cabildo Mayor Emberá Katío del Alto San Jorge (Córdoba).

50 Edinson Arley Bolaños, “¿Por qué la minga indígena resiste tanto tiempo?”, El Espectador, Bogotá, 28 de marzo, 2019. https://www. elespectador.com/colombia2020/territorio/por-que-la-minga-indigena-resiste- tanto-tiempo-articulo-857809 (22/7/2019).

51 Wirpsa, Rothschild y Garzón, “El poder del bastón”, 293.

52 Ariel Ávila, "Duque y la minga: paro nacional del 21 de octubre", Semana, Sábado, 17 octubre 2020. https://www.semana.com/ opinion/articulo/duque-y-la-minga-paro-nacional-del-21-de-octubre/202059/

53 Wirpsa, Rothschild y Garzón, “El poder..., 294.

54 Bolaños, “¿Por qué la minga indígena resiste tanto tiempo?”. ?”. El Espectador., Bogotá, 28 de marzo, 2019. https://www.elespectador. com/colombia2020/territorio/por-que-la-minga-indigena-resiste-tanto-tiempo-articulo-857809 (22/7/2019). 
-Y la Minga de marzo de 2019.

Lo que podemos inferir es que las luchas que hace veinte años inspiraron los movimientos colectivos están por consolidarse, si bien han logrado adelantos muy importantes en los contextos jurídico y político, y podríamos decir que, ante un avance sobreviene una retrogradación, como se puede ver en el siguiente informe fechado en 2005, para comparar:

En mayo de 2005, el Centro de Monitoreo de Desplazamiento Interno del Consejo Noruego para los Refugiados, citando al relator especial de las Naciones Unidas para los Pueblos Indígenas, advirtió que la violencia, la fumigación aérea de las plantaciones de coca y el desplazamiento ponían a los pueblos indígenas "en serio peligro de extinción".

A pesar de estos desafíos, los pueblos indígenas de Colombia han defendido durante siglos su autonomía, su vida, sus derechos, su cultura y su territorio. También han buscado alternativas locales, regionales, nacionales e internacionales para la coexistencia pacífica, la no violencia y la sostenibilidad medioambiental y económica $^{55}$.

Pareciera ser que la historia de los pueblos indígenas colombianos es una constante resiliencia frente a las violencias física, política, sociocultural, ambiental y económica; los resguardos indígenas están en un gran estado de vulnerabilidad.

La gravedad del asunto es que "de los 51 bloques petroleros, 37 se traslapan con 81 resguardos indígenas, principalmente en los departamentos de Putumayo y Caquetá. Esos contratos (2 TEA, 28 exploración y 7 producción) están en las manos de 12 empresas. Allí figuran Ecopetrol S.A., Gran Tierra Energy Colombia Ltd, Emerald Energy, Metra Petroleum Corp y Amerisur Exploración Colombia, entre otras. En total son más de 4'900.000 hectáreas..$^{56}$

Las políticas de Estado parecieran seguir amenazando las conquistas de los pueblos indígenas, pues se sigue ofertando un plan de desarrollo que fortalece más al sector financiero y a las multinacionales que a la preservación de los derechos conquistados por las comunidades indígenas. El desplazamiento se ha hecho presente también por la intromisión en las comunidades de grupos armados que traen consigo no solo amenazas a la población sino el asesinato de líderes y defensores de derechos humanos en los territorios, fenómeno que ya empieza a desplazar gota a gota a toda una población ${ }^{57}$.

55 Wirpsa, Rothschild y Garzón, “El poder del bastón”, 295.

56 El Tiempo, "Hay 81 resguardos indígenas en riesgo por 37 contratos petroleros", Bogotá, 26 de julio, 2019. https://www.eltiempo. com/vida/medio-ambiente/resguardos-indigenas-y-petroleo-en-colombia-380560. La redacción Mediambiente de este diario se basó en un estudio que hace parte de un proyecto llamado "Coalición para la promoción de los derechos territoriales de pueblos indígenas amazónicos y la protección del medio ambiente”, de la Organización Nacional de los Pueblos Indígenas de la Amazonia Colombiana (OPIAC), la Asociación Ambiente y Sociedad (AAS) y el Centro de Estudios para la Justicia Social Tierra Digna, con el apoyo de Rainforest Foundation Norway.

57 Quintero Alzate, "Los asesinatos de líderes en Colombia”. 
Los reclamos que han motivado las negociaciones que plantea el movimiento indígena colombiano se pueden sintetizar en el pliego de exigencias, los mismos requerimientos del 201358; veamos:

a. Territorio y tierras; seguridad jurídica de los territorios indígenas; ejercicio pleno del derecho al territorio; constitución, ampliación y saneamiento de los territorios indígenas;

b. Consulta previa sobre proyectos minero-energéticos e hidrocarburos; revocatoria directa de las concesiones o contratos de explotación minera en territorios indígenas;

c. Ejercicio de autonomía y gobierno propio; autonomía territorial; la cosmovisión y la autonomía en el ejercicio de gobierno propio;

d. Tratados de Libre Comercio y política agraria; promoción del referendo para la derogatoria de los TLC o la renegociación de los mismos; derogatoria de todas las normas que afecten la soberanía alimentaria;

e. Derechos humanos y aplicación del Acuerdo Final de Paz; respetar la autonomía de los Derechos Humanos de los pueblos indígenas; desmilitarización de los territorios indígenas; desmonte de los planes de consolidación y respeto al ejercicio de control territorial a través de la guardia indígena.

Recordamos los compromisos en el marco del Acuerdo Final de Paz, y que retomamos para su compresión, especialmente en el Capítulo Étnico del Acuerdo Final para la Terminación del Conflicto y la Construcción de una Paz Estable y Duradera, en el Sexto Acuerdo establece: Se creará una Instancia Especial de Alto Nivel con Pueblos Étnicos para el seguimiento de la implementación de los acuerdos, que se acordara entre el Gobierno Nacional y las FARC-EP y las organizaciones representativas de los pueblos étnicos.

- La instancia tendrá las funciones de actuar como consultora, representante e interlocutora de primer orden de la Comisión de Implementación, Seguimiento y Verificación del Acuerdo Final para la Paz y de Resolución de Diferencias (CSVR).

- Lo anterior sin detrimento de las funciones y atribuciones que tengan instancias de gobierno propio y de participación que ya existen.

- Las fuentes de financiación para la implementación de los acuerdos no involucrarán aquellos acuerdos en materia presupuestal que ya se hayan realizado entre el Gobierno Nacional y los pueblos indígenas y afrocolombianos, consignados en el Plan Nacional de Desarrollo Vigente y demás políticas consultadas y concertadas. ${ }^{59}$

La creación del Capítulo Étnico en el Acuerdo Final se convierte en un referente inédito en cuanto a garantías para la participación de las comunidades y pueblos étnicos, en un proceso de paz llevado a cabo entre un Estado y un grupo insurgente.

58 Fernando Dorado, "Las complejidades de la Minga Indígena y Popular", América Latina en movimiento, 31 de octubre (2013) https:// www.alainet.org/es/active/68655 (15/9/2019).

59 "Acuerdo final para la terminación del conflicto y la construcción de una paz estable y duradera" (2016), http://www.altocomisionadoparalapaz.gov.co/procesos-y-onversaciones/Documentos\%20compartidos/24-11-2016NuevoAcuerdoFinal.pdf (10/6/2019). 
En el perfil prospectivo del presente trabajo proponemos se incorpore el concepto de Modernidad Alternativa al tratar el tema de las comunidades indígenas. Dicho concepto es retomado de Gledhill, cuando se refiere a las llamadas "comunidades de resistencia" y a las "culturas de resistencia" que están en su base, para señalar que toda dominación trae consigo múltiples transformaciones de las formas dominantes y prácticas populares de resistencia que operan en la cotidianidad ${ }^{60}$. La interiorización de la dominación a través de las "culturas de resistencia" traducidas en los movimientos populares, pese a sus periodos de calma y sus contradicciones internas, han tenido - históricamente- resultados; en esa cultura de resistencia se anida la resiliencia ancestral.

El concepto de Modernidad Alternativa toma partido dentro de la polarización de los expertos en indigenismo.

[...] los intelectuales indigenistas estaban divididos entre aquellos que se interesaban en encontrar los medios para una integración exitosa de los indígenas en la Nación, y los que no veían con buenos ojos esta integración, considerándola como una fuente de desgracia para ellos mismos. Estos dos enfoques recuerdan el debate de gran actualidad sobre el grado y la manera en que los indígenas deben integrarse a la modernidad. Aquí hay interrogantes sobre la educación que deben seguir, la presencia de los servicios de salud al estilo occidental en sus comunidades, los procesos de interculturalidad, etc. Estos debates suelen referirse, en el fondo e implícitamente, a nociones de pureza y autenticidad (Sarrazin, 2015) ${ }^{61}$, ya que algunas personas asumen que la integración de los indígenas en la sociedad moderna puede "destruir" estas culturas. ${ }^{62}$

Pensamos que la minga, en cuanto imaginario social indígena, hace parte de esa Modernidad Alternativa que ha sido empoderada como andamio mental en la cultura de resiliencia indígena, y ha servido para expresar sus desencantos, pero al mismo tiempo para afianzar sus propias instituciones que han sobrevivido en la cultura de resistencia ${ }^{63}$.

\section{CONCLUSIONES}

Podemos decir que la Minga como imaginario social ha logrado macerar la mentalidad de grupo en las comunidades indígenas en Colombia, y desarrollar el espíritu de solidaridad fundamental para una Pedagogía de Resiliencia, en aras de lograr las luchas sociales y culturales. Ante el debate surgido para sobre

60 John Gledhill, El poder y sus disfraces. Perspectivas antropológicas de la política (Barcelona: Edicions Bellaterra, 2000$), 308$.

61 El texto citado remite a Jean Paul Sarrazin, “The Protection of Cultural Diversity: Reflections on its Origins and Implications”, Justicia, n. ${ }^{\circ} 27$ (2015). Doi: http://doi.org/10.17081/just.3.27.32

62 Jean Paul Sarrazin, "La categoría indígena definida desde la hegemonía y sus alcances en la institucionalidad colombiana”, Justicia, n. 32 (2017): 149. Doi: https://doi.org/10.17081/just.23.32.2909

63 Bolaños, “PPor qué la minga indígena resiste tanto tiempo?. (Bogotá: El Espectador, 2019) https://www.elespectador.com/ colombia2020/territorio/por-que-la-minga-indigena-resiste- tanto-tiempo-articulo-857809 (22 de Julio de 2019 ). 
cuál es el aporte de las comunidades indígenas al PIB de la nación, es bueno recordar una interpretación desde la visión del Nuevo Constitucionalismo Latinoamericano, según la cual se debe reconocer su aporte en la preservación de la biodiversidad, un aspecto muy reconocido en el marco del giro decolonial. En América Latina esa visión ha transitado hacia el biocentrismo, o denominado giro biocéntrico. Esos conceptos han sido empoderados por la Minga en sus luchas; es una ecociudadanía activa que busca recuperar su cultura ancestral, los conceptos de derechos de la Naturaleza y el derecho de los animales. Los saberes tradicionales, al ser reivindicados como sujetos con derecho, y en especial la naturaleza se encuentra como uno de los logros más importantes. De esa manera, la tierra como Pachamama, ofrece un contexto para las políticas y la gestión ambiental basado en la buena vida (sumak kawsay). Es la pedagogía de la desaceleración que proponemos en etapa postpandemia, la pedagogía apofática.$^{64}$ Con la experiencia de la pandemia covit19 se ha podido reivindicar que el aporte de la Minga al giro biocéntrico es de la mayor importancia. La ecología política y la ética ambiental, revisan los impactos del concepto de valor intrínseco y se presenta una serie de desafíos futuros en el terreno de la política y la gestión; abriendo las puertas a nuevas formas de valoración ambiental y articulación con los saberes indígenas.

Los imaginarios sociales tienen sus propias representaciones y puede ser recreados a partir de sus líderes, no sólo los que son protagonistas en su momento, sino aquellos que la imaginería popular los va creando. Históricamente podemos recordar ejemplos de resiliencia indígenas desde Agustín Agualongo (1780-1824); Pedro Pascasio Martínez, el adolescente exaltado en la Batalla de Boyacá por su principio de honestidad (1807-1885) hasta Quintín Lame (18801967). La historia de los imaginarios sociales de resiliencia indígena tiene una historia no contada todavía. La historiografía positivista negó la participación de las comunidades indígenas en la historia, y eso lo documenta Heraclio Bonilla; "la historia de la historiografía de la independencia pasó por varios momentos (...) Por cierto que, en esas historias, los indios (...) no aparecían ni en los pies de página." ${ }^{15}$ Debemos reconocer que el proceso de independencia no reconoció suficientemente su condición social, pues si bien es verdad desde la Constitución de Tunja (1811) se le reconoció como ciudadanos a todos los mayores de 14 años $^{66}$, no se les reconoció su condición de pueblos indígenas; siguieron siendo excluidos por las elites de los libertadores. Sintetizamos los aportes de la Minga como imaginario social en los siguientes puntos:

a. La minga, como forma de organización de las culturas indígenas, permite recuperar una pedagogía de resiliencia para la paz; la transmodernidad en el marco del giro decolonial potencia los derechos sociales de los movimientos

64 José Pascual Mora García, La pedagogía apofática, una nueva pedagogía de la desaceleración ante el Covid-19. Heurística, nro 80 (2020). http://erevistas.saber.ula.ve/index.php/educere/article/view/16475 (20 de julio de 2020).

65 Heraclio Bonilla, La construcción del conocimiento histórico: Errata y bricolaje de la historia. (Lima: Instituto de Estudios Peruanos, 2010), 15.

66 Jorge Patiño Rojas, República de Tunja 1811-1816, Análisis Constitucional.. 
indígenas en Colombia. La ecociudadnía activa propone una lucha que invoca la paz y no la violencia como estrategia para alcanzar sus reivindicaciones sociales.

b. Contextualizamos el tema de la minga señalando que, en los últimos veinte años, se han realizado más de cincuenta manifestaciones sociales, pero, sobre todo, destacamos la manifestación de imaginarios sociales de los pueblos indígenas de Colombia.

c. La minga sigue siendo un instrumento para exigir las reivindicaciones sociales, reconocimientos de sus derechos democráticos, de sus iniciativas frente a los acuerdos de paz, y luchas geográficas de la tierra, la autonomía de la justicia, y la justicia transicional.

\section{REFERENCIAS}

"Acuerdo final para la terminación del conflicto y la construcción de una paz estable y duradera". 24 de noviembre de 2016. http://www.altocomisionadoparalapaz.gov.co/procesos-y-conversaciones/Documentos\%20compartidos/24-11-2016NuevoAcuerdoFinal.pdf (10/6/2019).

Ávila, Ariel, "Duque y la minga: paro nacional del 21 de octubre", Semana, Sábado, 17 octubre 2020. https://www.semana.com/opinion/articulo/duque-y-la-minga-paro-nacional-del-21de-octubre/202059/

Amselle, Jean-Loup. “En nombre de los pueblos: primitivismos y poscolonialismos". Revista Colombiana de Antropología 49, n. 2 (2013): 207-221.

Báez-Jorge, Félix. "Antropología e indigenismo en Latinoamérica: señas de identidad". En Motivos de la antropología americanista. Indagaciones en la diferencia, coordinado por Miguel León-Portilla. México D. F.: Fondo de Cultura Económica, 2001, 424-444.

Bolaños, Edinson Arley. “¿Por qué la minga indígena resiste tanto tiempo?”. El Espectador., Bogotá, 28 de marzo, 2019. https://www.elespectador.com/colombia2020/territorio/por-que-la-minga-indigena-resiste-tanto-tiempo-articulo-857809 (22/7/2019).

Bonilla, Heraclio, La construcción del conocimiento histórico: Errata y bricolaje de la historia. (Lima: Instituto de Estudios Peruanos, 2010)

Simón Bolívar, Obras Completas (Caracas: Pool Reading, 1975)

Brubaker, Rogers. Ethnicity without groups. Cambridge: Harvard University Press, 2014.

De Sousa Santos, Boaventura. Para una democracia de alta intensidad. Renovar la teoría crítica y reinventar la emancipación social. Buenos Aires: Clacso, 2006

http://bibliotecavirtual.clacso.org.ar/ar/libros/edicion/santos/Capitulo\%20III.pdf

Dorado, Fernando. "Las complejidades de la Minga Indígena y Popular". América Latina en movimiento, 31 de octubre (2013). https://www.alainet.org/es/active/68655 (15/9/2019).

Dussel, Enrique. "Transmodernidad e Interculturalidad. (Interpretación desde la Filosofía de la Liberación)". México: UAM-Iz., 2005. http://red.pucp.edu.pe/ridei/wp-content/uploads/ biblioteca/090514.pdf.

El Espectador (Editorial). “Los siguen matando, cuando son más necesarios", Bogotá, 28 de mayo, 2020, http://elespectador.com/opinion/editorial/los-siguen-matando-cuando-son-mas-necesarios-articulo-921437 (30/05/2020).

El Tiempo. "Siete militares habrían violado a niña embera de 13 años", Bogotá, 24 de junio, 2020, https://www.eltiempo.com/justicia/investigacion/siete-militares-habrian-violado-a-nina-embera-510446 (30/05/2020).

El Tiempo. "Hay 81 resguardos indígenas en riesgo por 37 contratos petroleros", Bogotá, 26 de julio, 2019, https://www.eltiempo.com/vida/medio-ambiente/resguardos-indigenas-y-petroleo-en-colombia-380560 (30/05/2020).

Fanon, Frantz. Los condenados de la tierra. México, D.F.: Fondo de Cultura Económica, 1961.

Fernández Retamar, Roberto. Todo Caliban. Bogotá: Editorial ILSA, 2005. 
Findji, María Teresa. “From Resistance to Social Movement: The Indigenous Authorities Movement in Colombia". En The Making of Social Movements in Latin America: Identity, Strategy, and Democracy, editado por Escobar Arturo y Sonia E. Álvarez. New York: Westview Press, 1992, 112-133.

Gledhill, John. El poder y sus disfraces. Perspectivas antropológicas de la política. Barcelona: Edicions Bellaterra, 2000.

Gros, Christian. Colombia indígena: Identidad cultural y cambio social. Bogotá: CEREC, 1991.

Gutiérrez Ramos, Jairo. Los indios de Pasto contra la República (1809-1824). Bogotá: Instituto Colombiano de Antropología e Historia, 2007.

Halbwachs, Maurice. Les cadres sociaux de la mémoire. París: Gallimard, 1994.

Hobsbawm, Eric. "Entrevista sobre el siglo XXI. Las predicciones de un perspicaz historiador", 20 de septiembre (2004). https://www.lainsignia.org/2004/septiembre/cul_061.htm (11/10/2019).

Hobsbawm, Eric y Terence Ranger, editores. La invención de la tradición. Barcelona: Editorial Crítica, 2003.

Langebaek Rueda, Carl Henrik. Arqueología colombiana. Ciencia, pasado y exclusión. Bogotá: Colciencias, 2003.

Laurent, Virginie. Comunidades indígenas, espacios políticos y movilización electoral en Colombia, 19901998. Motivaciones, campos de acción e impactos. Bogotá: Institut français d'études andines / Instituto Colombiano de Antropología e Historia-ICANH, 2005.

Marquardt, Bernd. Historia del derecho de Hispanoamérica: En perspectiva transnacional y socio-cultural. Bogotá: Casa Editorial Ibáñez, 2019.

Mora García, José Pascual. "Aproximación a las pedagogías alternativas: de la pedagogía de la diversidad a las pedagogías de la resiliencia en el marco del postacuerdo". Revista Historia de la Educación Colombiana 22, n. 22 (2019): 39-66. https://doi.org/10.22267/rhec.192222.51

Mora García, "La pedagogía apofática, una nueva pedagogía de la desaceleración ante el Covid19." Heurística, no 80 (2020).

http://erevistas.saber.ula.ve/index.php/educere/article/view/16475 (20 de julio de 2020).

Mora García, José Pascual. “Mirada sinóptica sobre la Constitución Social a propósito del Centenario de las Constituciones Sociales de Weimar (1919), Querétaro (1917) y el proceso constitucional colombiano". Heurística, 23 (2020) (En proceso de edición)

Mora García, "La construcción del campo intelectual de la historia de la educación y su aporte a la paz pedagógica: Una mirada interdisciplinaria. Derecho y Realidad, 17(33). (2019) https:// doi.org/10.19053/16923936.v17.n33.2019.10096.

Mora García, José Pascual. Pedagogías, Paz y Poblaciones Resilientes en el marco del Postacuerdo colombiano. Tunja: UPTC (Trabajo final del postdoctorado en proceso de edición). 2020

Patiño Rojas, Jorge. República de Tunja 1811-1816. Análisis constitucional. Tunja: Búhos editores Ltds. 2019.

Perry, Jean Paul. “La exposición arqueológica y etnográfica de 1938 en Colombia: Un primer intercambio cultural". En Arqueología y Etnología en Colombia. La creación de una tradición científica, compilado por Carl Henrik Langebaek y Clara Isabel Botero. Bogotá: Universidad de los Andes, Centro de Estudios Socioculturales, 2009, 79-94.

Pineda Camacho, Roberto. Estado y pueblos indígenas en el siglo XX. La política indigenista entre 1886 y 1991. Revista Credencial Historia, n. ${ }^{\circ} 146$ (2016). http://www.revistacredencial.com/ credencial/historia/temas/estado-y-pueblos-indigenas-en-el-siglo-xix (9/7/2019).

Pineda Camacho, Roberto. "La Constitución de 1991 y la perspectiva del multiculturalismo en Colombia". Revista Alteridades 7, n. ${ }^{\circ} 14$ (1997): 107-129.

Publimetro. "Revelan audio de funcionario de MinInterior insultando a indígenas caucanos", Bogotá, 21 de mayo, 2020. https://www.publimetro.co/co/noticias/2020/05/21/odio-hijueputas-funcionario-mininterior-indigenas-del-cauca.html (22/5/2020).

Quintero Alzate, María Fernanda. “Los asesinatos de líderes en Colombia: ¿un tema nacional o internacional?". América Latina en movimiento, 5 de julio (2019). https://www.alainet.org/es/ articulo/199768 (5/9/2019).

Revéiz, Edgar. Cooptación o democracia. La transgresión moral de las élites y el sometimiento de los estados. (Bogotá: Academia colombiana de ciencias económicas, 2015. 
Salazar Ugarte, Pedro. "El nuevo constitucionalismo latinoamericano (una perspectiva crítica)". En El constitucionalismo contemporáneo. Homenaje a Jorge Carpizo, coordinado por Luis Raúl González Pérez y Diego Valadés. México: UNAM, 2012, 345-387.

Sañudo Pazos, María Fernanda. Tierra y género: Dilemas y obstáculos en los procesos de negociación de la política de tierras en Colombia. Bogotá: Editorial Pontificia Universidad Javeriana, 2015.

Sarrazin, Jean Paul. "La categoría indígena definida desde la hegemonía y sus alcances en la institucionalidad colombiana". Justicia, n. 32 (2017): 139-159.

Sarrazin, Jean Paul. "The Protection of Cultural Diversity: Reflections on its Origins and Implications". Justicia, n. ${ }^{\circ} 27$ (2015): 99-117.

Sarrazin, Jean Paul. "Avatares del pluralismo: Usos y abusos del concepto de Diversidad Cultural en medios institucionales". Principia Iuris 13, n. ${ }^{\circ} 26$ (2016): 55-73.

Silva Rodríguez, Paula, y Camilo Ramírez Gutiérrez. "Fórmulas para la paz: eficacia de los derechos humanos en las zonas de violencia y los campos sociales minados por el capitalismo global". En Paz a través del derecho y de la constitución, coordinado por Bernd Marquardt, David Ernesto Llinás Alfaro y Aura Helena Peñas Felizzola. Bogotá: Grupo Editorial Ibáñez, 2016.

Suescún M., Armando. Derecho y sociedad en la historia de Colombia: El derecho chibcha. Siglo IX - Siglo XVI. Tunja: Universidad Pedagógica y Tecnológica de Colombia, 1998.

Walsh, Catherine, editora. Pedagogías decoloniales, prácticas insurgentes de resistir, (re)existir, y (re)vivir. Quito: Ediciones Abya-Yala, 2013.

Wirpsa, Leslie, David Rothschild y Catalina Garzón. “El poder del bastón. La resistencia indígena y la construcción de la paz en Colombia". En Colombia: La construcción de la paz en tiempos de guerra, editado por Virginia M. Bouvier. Bogotá: Editorial Universidad del Rosario, 2014, 293-315.

Cómo citar:

Mora García, José Pascual; Correa Alfonso, José del Carmen. “La minga como imaginario social. Una mirada a la pedagogía de resiliencia indígena en Colombia". Revista Historia de la Educación Latinoamericana. 22 No. 35 (2020): 163-180 https://doi.org/10.19053/01227238.10355

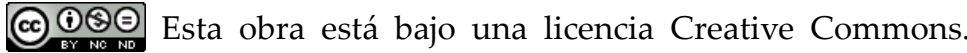
Reconocimiento-No Comercial-Sin Obra Derivada 2.5 Colombia. 